extension of the tumor cells through the sclera-the sclera, by the way, is a tissue in which any invasion by foreign cells is easily found when microscopic examination is made-along the sheath of one of the ciliary nerves.

And herein is the case instructive. Sections of the eyeball should have been made as soon after the enucleation as possible. Had this been done the extension into the orbital tissue could have been recognized, prompt exenteration of the orbit could have been done, and the patient thus spared the dangers incident to the further growth of the tumor.

I think this one case demonstrates the following proposition: When enucleation of the eyeball is done for uveal sarcoma, the globe, if it has no gross signs of extension externally, should be sectioned, as soon as it can be prepared, and examined for possible microscopic extension to the orbital tissue. It follows, of course, that if such extension is found exenteration of the orbit should be done immediately.

\section{A NEW COMBINED OPERATING AND OFFICE TABLE.}

JOHN H. DOWNEY, M.D. GAINESVILLE, GA.

I have devised a new combination operating, office and fracture table, in which I have tried to arrange the traction in every desired direction that will hold the patient in a partially suspended and yet comfortable position. It gives the physician free access to his patient and allows him to apply a dressing or a plasterof-Paris splint to any part of the body.

In Figure 1, the table is arranged for general surgery ; it is just as good as any for general operating and can be easily arranged for office work. By dropping the lower portion of the top and placing the leg rests and stirrups in position it can be used for special operations about the vagina, uterus or rectum.

Figure 2, shows the arrangement for treatment of fracture of the femur or fracture of the upper two-

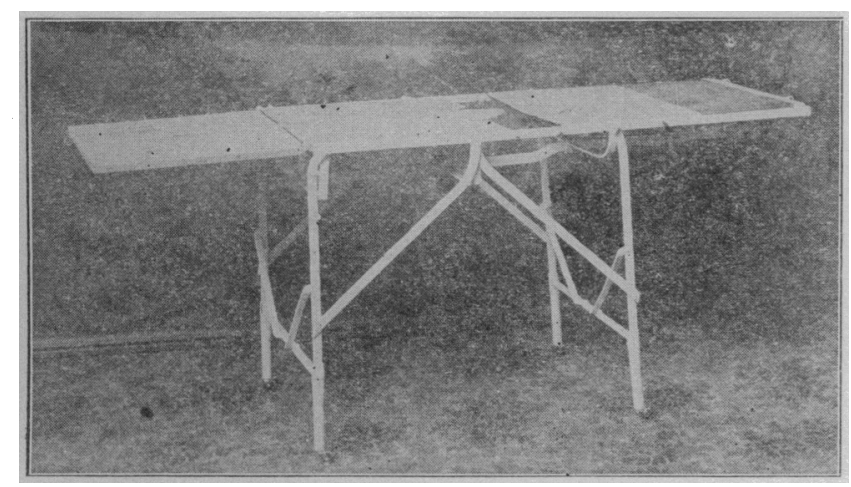

thirds of the tibia and fibula. In the latter the whirl arch is thrown forward and locked, as in Figure 5, traction being made against the head of the tibia, the foot being fastened to the foot plate as in Figure 3 . When the lower thirds of these bones are the site of fractures the whirl arch is thrown backward on the same plan as the top of the table and the extension is made in a straight direction. In this fracture the cast need not go above the knee; so only two sections are necessary; first, the slipper, after this sets, put on the strap, apply extension until fragments are in opposition (the center post makes the counter extension) then apply the upper section of the cast to just where the knee makes the bend. The fixation points are the heel and instep of the foot, the tapering calf and the head of the tibia. In fractures of the upper part of the tibia it is necessary to carry the plaster above the knee and the cast has to be applied in three sections ; first, the slipper, as mentioned above, then an elbow at the knee, leaving the site of the fracture bare. To do this, the foot is fastened to the foot plate and the whirl arch thrown forward; as in Figure 4, the leather strap is reversed so as to grasp the head of the tibia and to make the exten-

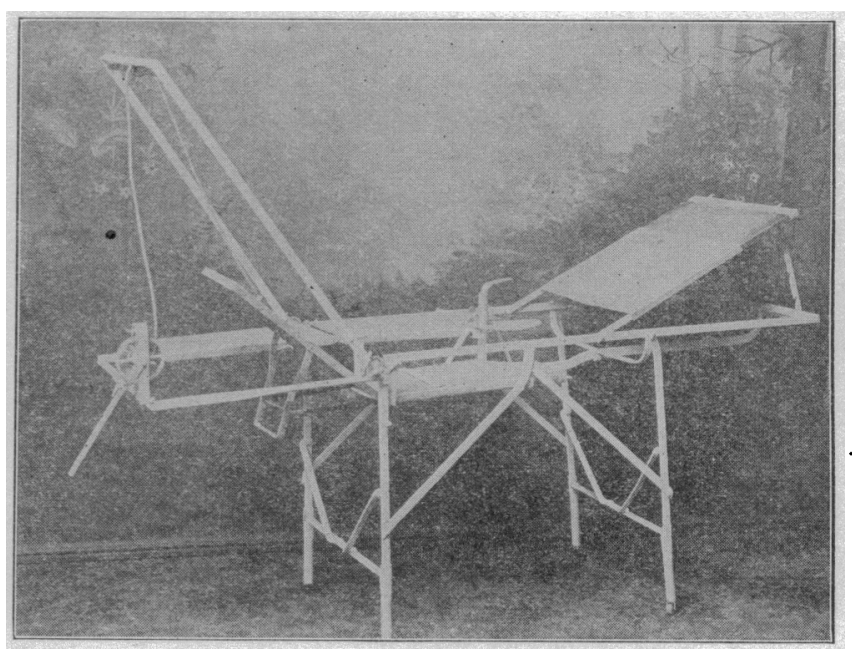

sion upward and forward-the foot plate making counter extension downward and backward. When the fracture is properly adjusted, the middle section of the cast is applied.

In fractures of the femur, the patient's leg is fixed in the proper position and the first section of the cast is applied in the usual way to just above the knee, making the cast a little heavy just below the knee to prevent denting during extension. When this section is hard, which only takes a few minutes, the patient is placed on the table as shown in Figure 3, and the knee strap is applied so as to grasp the head of the femur; the necessary extension is made, the center post being the counter extension, the fragment is adjusted and the upper section of the cast is then applied, begin. ning at the upper end of the lower section by spreading a small amount of plaster for the new starting point and towake the weld firm; carry the plaster up to the ensiform cartilage, making the necessary layers to give strength, reinforce it at the hip and at the junction of the two sections, and you have the double angular splint.

Note how perfectly extension and counter-extension can be made and how slowly and steadily extension is made, giving the muscles time to relax. When the fragments are in position there is no giving way and no slipping out of place. The operator has perfect access to the patient without the slightest unnecessary movement. Perfect fixation is afforded by the abrupt angles at the knee and hip, making shortening and deformity impossible. The fracture must unite as it has been placed, there is no eversion or inversion, there is freedom of every movement without pain as the fragments and every muscle connected with the fracture are fixed. The patient can attend the calls of Nature at any time in natural positions, he can sit or walk at will. There is no four or six weeks in bed, no weight or suspension apparatus to be adjusted, very little pain and no deformity. 
These look like broad statements, but they have been proved time and again.

In fracture of the clavicle arrange the table as in Figure 1 ; elevate the head, telescope it up to full extent, hook the spinal support in hole where the center post was taken out of place, place the patient on the table with the back of the head resting on the telescoped part, the spinal support holding the chest up, then carry the shoulders upward, outward and backward until the fractured end is in position.

Take an ordinary roller bandage and tie the shoulders

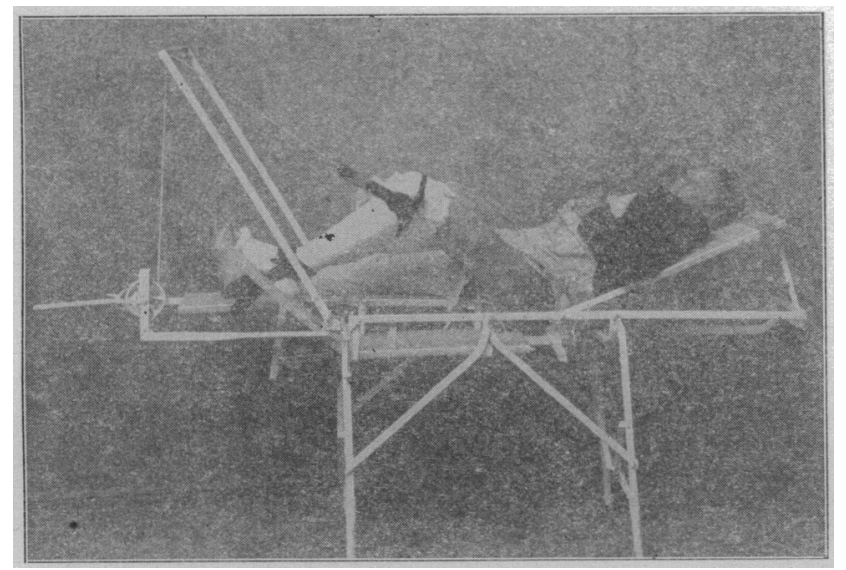

in this position to the sides of the table, cover the upper fourth of the chest with cotton wadding and hold this in place with a few turns of a roller bandage. Next take a strip of adhesive plaster two inches wide and long enough to reach from the trochanters up to the side of the axilla and two inches out under the arms, commence with the plaster roller at the lower part of the cotton wadding and go up to the axilla and make a double figure of eight back and front. At this point loosen the plaster under the arms and carry it down beside the

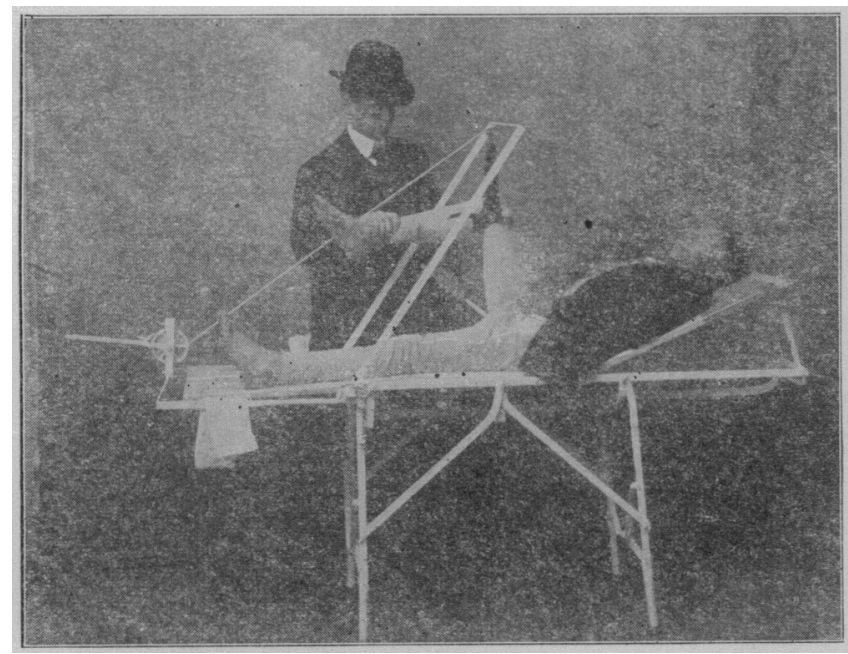

chest, making a loop at the top, and cover this with another layer of plaster. With this splint both shoulders and scapula are fixed, the sound shoulder acting as a fulcrum to hold the injured one up. The double figure of eight turns around the neck and both shoulders prevent the forward and downward drooping, and the adhesive plaster prevents the elevation of the shoulders, thus giving perfect fixation, while at the same time the appliance is comfortable and will allow the patient to rear his arm in his sleeve. The only other dressing necessary is a simple sling in which the hand may rest.
This is a simple, comfortable and efficient method of treating these fractures and the assistance of this table makes it easy of application as it gives easy access to the patient.

In dislocation of the hip, knee, ankle or shoulder, this table can be arranged to give the desired amount of cxtension in any direction; Figure 4 shows its application in backward dislocation of the hip, one man doing eficctually the work of three and with greater ease to himself and less pain to the patient. The finger in the center post holds the patient to the table, the ratchet pulls the bead back in the direction of the acetabulum, the side lever lifts the head from behind the rim and the right hand uses the leg as a crank to rotate the head into the socket. Figure 5 shows a device for suspending the patient with an injury or disease of the spine necessitating a plaster jacket. This is done by telescoping

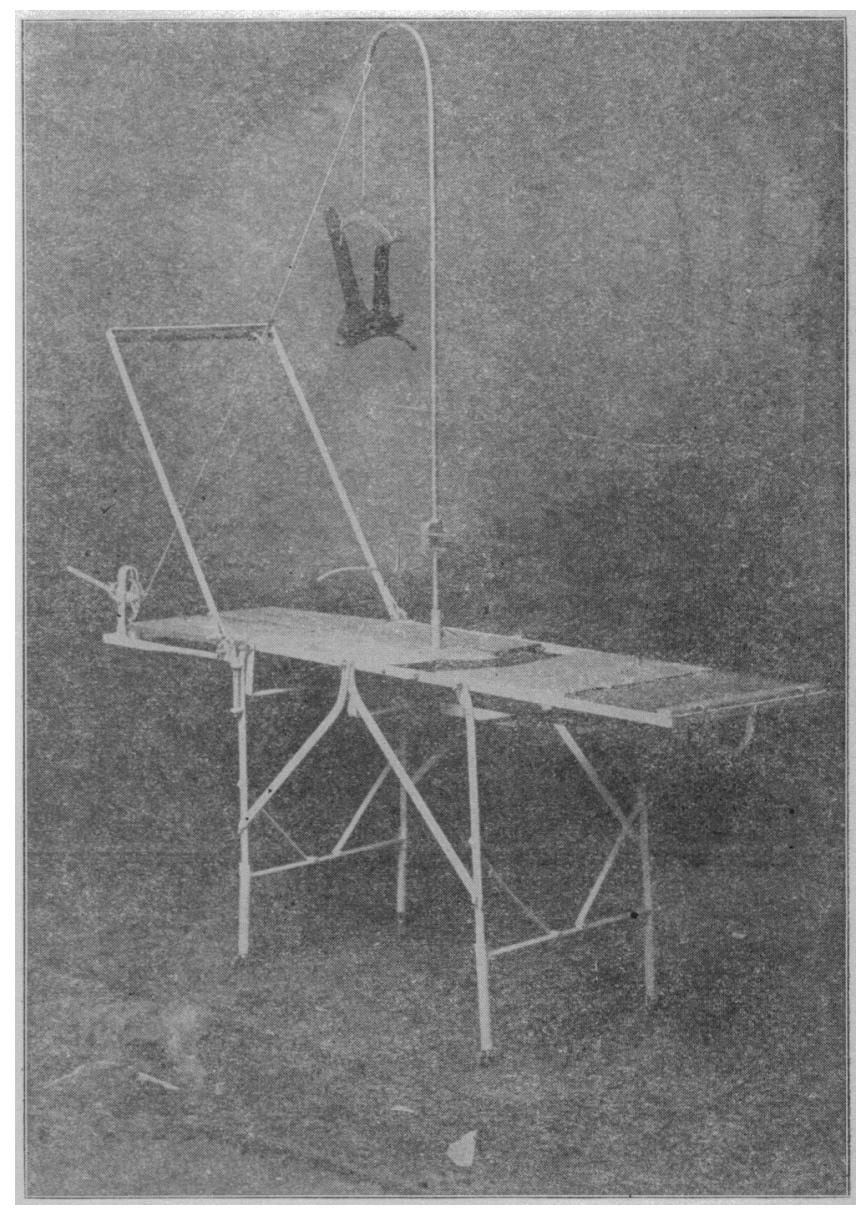

the head rest, with the patient sitting on the table, the legs dropping through the table are adjusted, the clamp holds the patient to the table, the leather cuff is lifted around the neck and drawn up by the ratchet to the desired point and locked. The patient is firmly fixed and the physician has access to every part without a single movement and any amount of traction can easily be kept until the plaster has thoroughly set.

Comfort in Winter Traveling.- The exhaust from the engine can be made to thoroughly heat the car, which can readily be made inclosed, and in zero weather the doctor can go to the bedside as warmly as he can sit in his surgery. Instead of getting thawed out at the stove before approaching the sick one, he is ready immediately to give attention to his patient.A utomobile. 